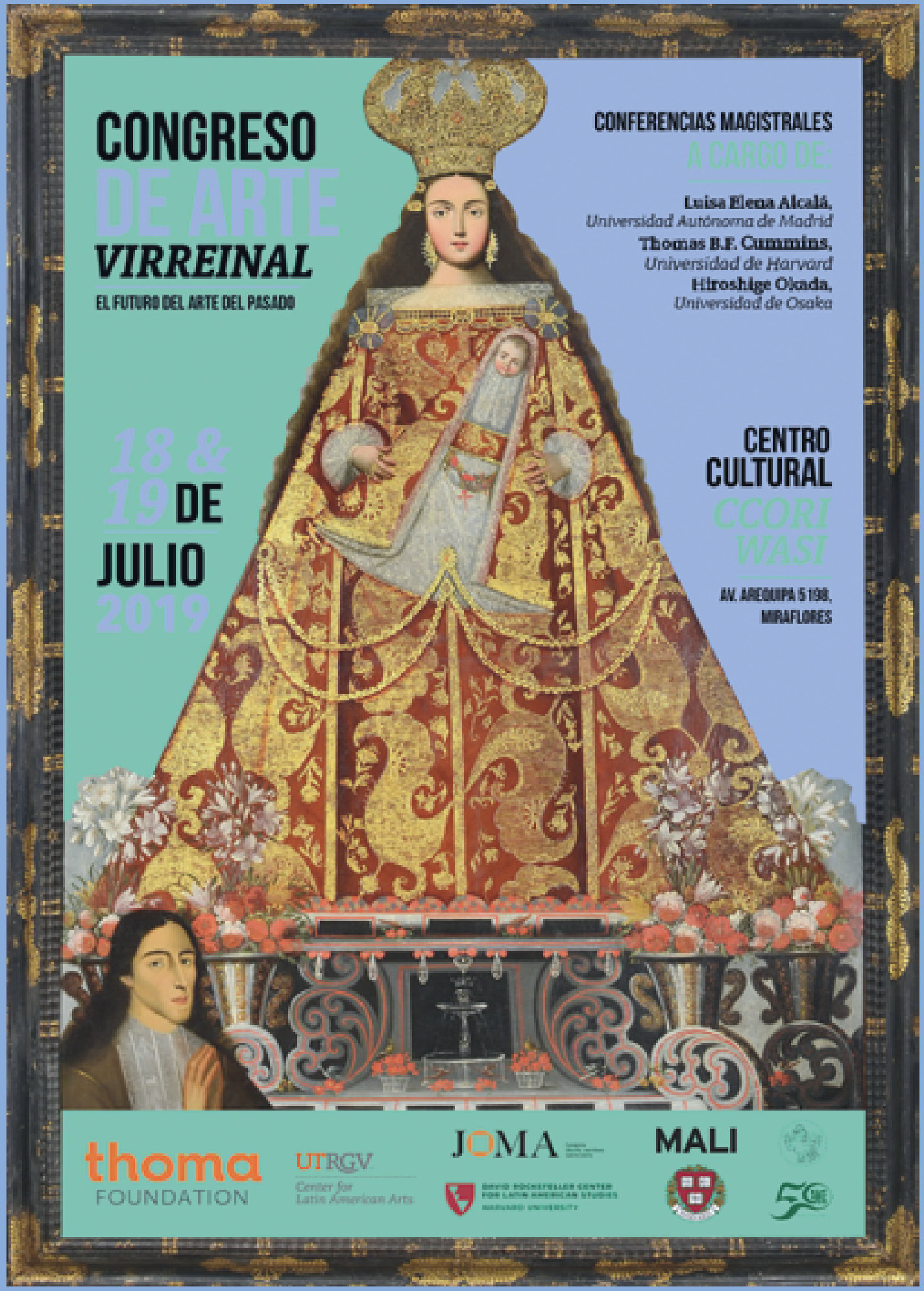




\section{Noticias 2019}
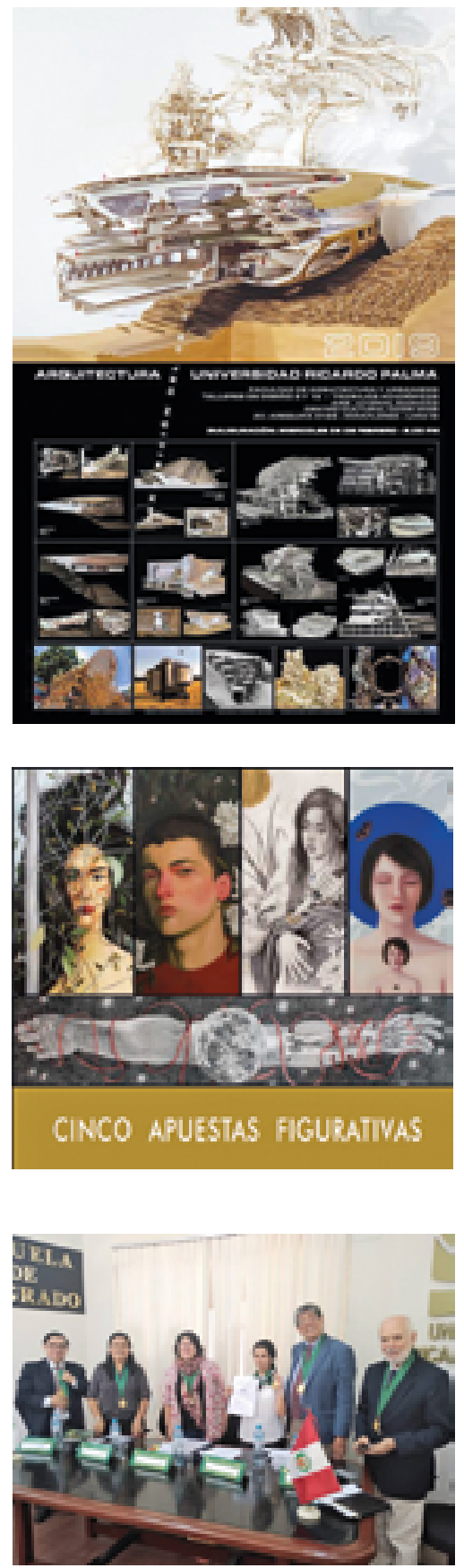

María Angélica Rozas Rozas Alvarez Instituto de Investigaciones Museológicas y Artísticas Universidad Ricardo Palma mrozas@urp.edu.pe

Como todos los años, el 6 de febrero se realizó en la Galería de Artes Visuales, la Exposición del Taller 5 y 15 de la Facultad de Arquitectura y Urbanismo de la Universidad Ricardo Palma, dirigido por el Arq. Juvenal Baracco.

Cinco apuestas figurativas, exposición colectiva de las artistas Gala Albitres, Karina Huertas, Gloria Quispe Tinco, Salima Black y Judith Vergara, en la que a través de dibujos, pinturas y grabados en diferentes formatos nos muestran representaciones de la naturaleza y lo real en donde sujetos y objetos aparecen encarnando cualidades simbólicas y acuciosos procedimientos técnicos que los hacen visibles. La exposición estuvo del 8 al 31 de marzo en la Galería de Artes Visuales de la Universidad Ricardo Palma.

El 4 de abril tuvo lugar la disertación de la tesis Propuesta de un Plan Museológico para el Museo Etnográfico Amazónico del Centro Cultural José Pio $A z a$, de María Angélica Rozas Rozas Álvarez, egresada de la maestría en Museología y Gestión Cultural. En la foto, de izquierda a derecha: Dr. Luis Alberto Vicuña, Mg. Magdalena Cruz, Dra. Rocío Trinidad Zapata, Mg. María Angélica Rozas Rozas Álvarez, Dr. Alberto Martorell Carreño y Dr. Alfonso Castrillón Vizcarra. 
Geografías geométricas, esculturas, muestra individual del arquitecto, escultor, ceramista y docente universitario Jaime Botteri Lequernaqué, conformada por dieciséis obras escultóricas divididas en dos series: "Relieves" y "Nudos" que tienen como común denominador la línea, el vibrante color y la forma geométrica con una precisión casi arquitectónica. La exposición estuvo del 5 al 28 de abril en la Galería de Artes Visuales de la Universidad Ricardo Palma.

El 30 de abril en el marco de las muestras "Otras historias posibles" (Museo de Arte de Lima) y "Diseño Gráfico Pioneros de los sesenta" (Galería de Artes Visuales) se llevó a cabo el conversatorio Diseño Gráfico-Pioneros de los años 60' y que estuvo a cargo de Alfonso Castrillón Vizcarra quien conversó con Carlos González, Víctor Escalante, Ciro Palacios, Octavio Santa Cruz y Jesús Ruiz Durand sobre la historia del diseño gráfico contemporáneo en el Perú. El evento se realizó en el Auditorio AFP Integra del MALI.

Diseño Gráfico Pioneros de los sesenta, muestra colectiva de los artistas Carlos González, Jesús Ruiz Durand, Claude Dieterich A., Octavio Santa Cruz, Ciro Palacios y Víctor Escalante. Muestra en la que se pudieron apreciar afiches, carátulas de libros y logotipos que si no siguen vigentes, han quedado como íconos del diseño gráfico en el Perú. La exposición estuvo del 4 al 27 de mayo en la Galería de Artes Visuales de la Universidad Ricardo Palma.

Testimonio de vida artística. Homenaje a la Bauhaus del artista plástico alemán Rolf Bertschat. Es un recuento de sus obras desde 1957 hasta la actualidad, y cuyo trabajo está marcado por los procesos sociales producto del impacto de la segunda Guerra Mundial y la escuela de la Bauhaus, simbolizado en la ausencia de la imagen humana y la represión de la sensibilidad del hombre bajo la guerra. La exposición estuvo del 1 al 30 de junio en la Galería de Artes Visuales de la Universidad Ricardo Palma.

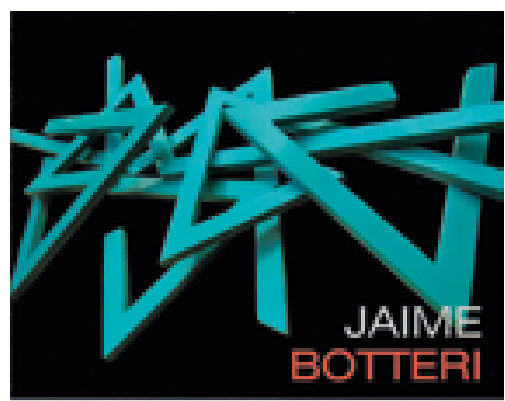

Goografias gocmetricas, esoulturas
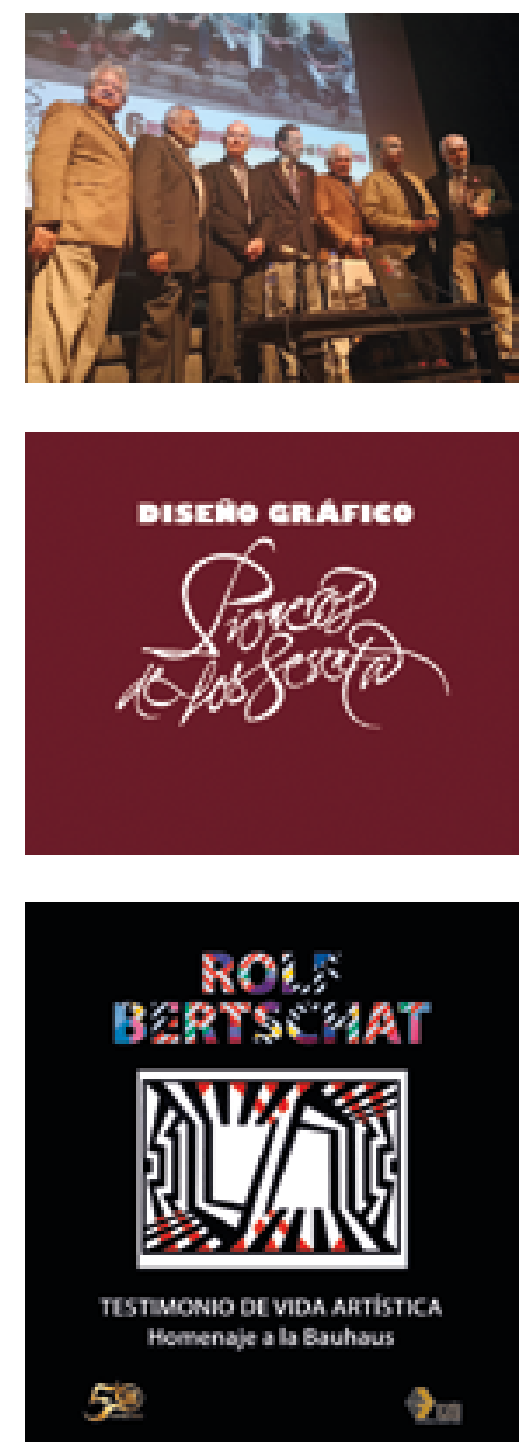


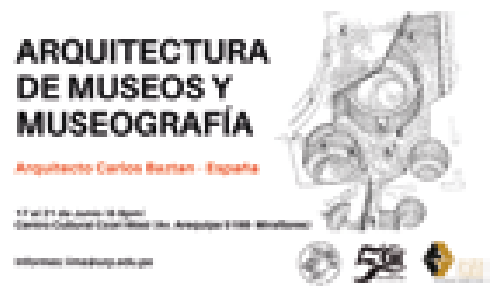

Del 17 al 21 de junio se realizó el curso de Arquitectura de Museos y Museografía a cargo del Arq. Carlos Baztán de Madrid, España, organizado por el Instituto de Investigaciones Museológicas y Artísticas de la Universidad Ricardo Palma. Donde se expuso a los participantes, los conceptos y la transmisión de ideas y ejemplos para gestionar la creación o renovación de un museo equilibrado y sostenible; poniendo énfasis en su arquitectura y sobre qué se debe reflexionar, por quien, en qué orden y con qué objetivos.

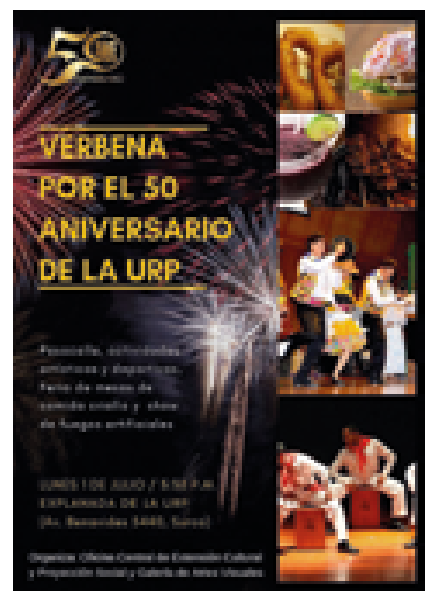

Se llevó a cabo el 1 de julio en el campus universitario de la Universidad Ricardo Palma, la verbena por el $50^{\circ}$ Aniversario de nuestra casa de estudios, evento que contó con pasacalles, actividades artísticas, feria de mesas de comida criolla y show de fuegos artificiales y que fue organizado por el Rectorado, la Oficina Central de Extensión Cultural y Proyección Social y la Galería de Artes Visuales.

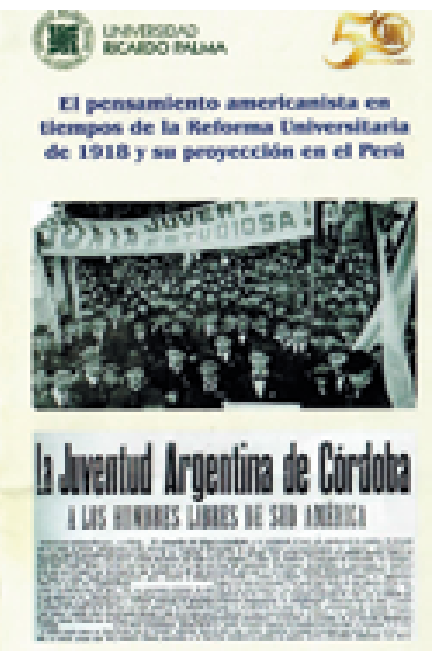

El Pensamiento Americanista en Tiempos de la Reforma Universitaria de 1918 y su Proyección en el Perú, exposición documental donde cuenta cómo la Reforma Universitaria de 1918 eclosionó en la ciudad argentina de Córdoba y fue la consecuencia de un proceso que al consolidarse, se convirtió en un hecho trascendente para la formación académica de las casas de estudio de América Latina. La muestra estuvo organizada por el Instituto de Investigación del Patrimonio Cultural de la Universidad Ricardo Palma y estuvo expuesta del el 4 al 29 de julio en la Galería de Artes Visuales de la Universidad Ricardo Palma. 
Del 8 al 12 de julio la Lic. Marta Miyashiro, dictó el "Taller de redacción académica con estilo APA" organizado por el Instituto de Investigaciones Museológicas y Artísticas de la Universidad Ricardo Palma. El taller trabajó la importancia de las publicaciones académicas, las normas internacionales que se usan en la redacción académica, los diversos tipos de párrafo y cómo estos se relacionan mediante conectores lógicos así como, las partes de un informe de investigación y de un artículo de revista.

El 17 de Julio, se presentó Las buenas intenciones, libro de Alfonso Castrillón Vizcarra, en el que reúne una serie de artículos dispersos en revistas y periódicos, o en catálogos de exposiciones, hoy difíciles de encontrar y que nos permiten ver con más claridad los temas culturales de estas dos últimas décadas. La presentación estuvo a cargo de Claudia Balarín, Christabelle Roca Rey y Luis Sihuacollo.

El 18 y 19 de julio se llevó a cabo el I Congreso de Arte Virreinal, El futuro del arte del pasado, evento que se realizó con el apoyo de Carl \& Marilynn Thoma Art Foundation (USA) Harvard University (USA), la Fundación Obra Pía José María Sancho Dávila (Perú) The Center for Latin American Arts (USA), el Museo de Arte de Lima; evento que fue organizado por Verónica Muñoz-Nájar (Universidad de California, Berkeley), Katherine McAllen (Universidad de Texas Rio Grande Valley) y el Instituto de Investigaciones Museológicas y Artísticas de la Universidad Ricardo Palma.
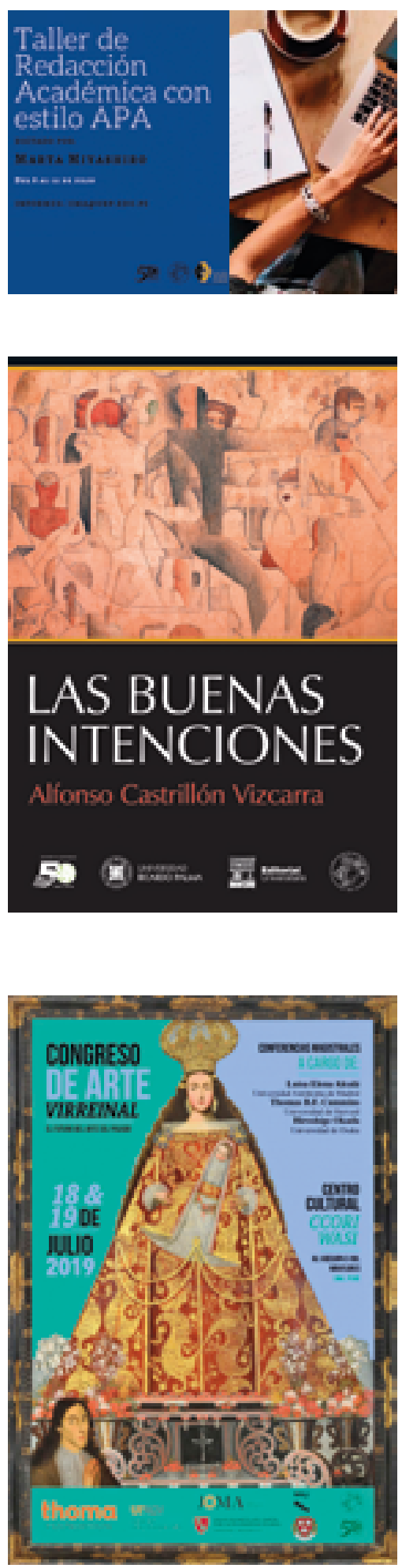

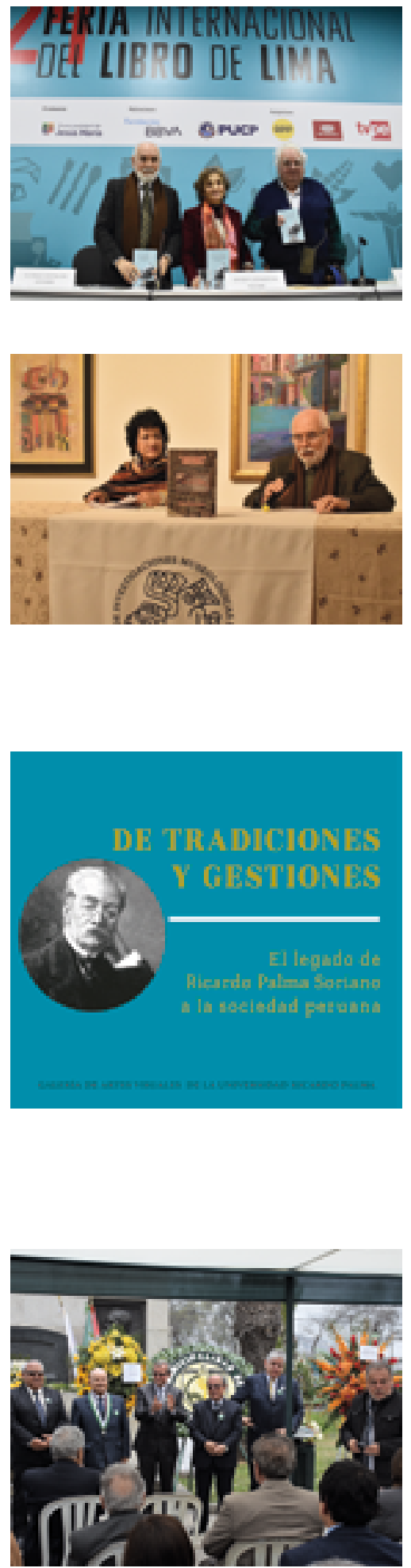

El 23 se julio en la 24 edición de la Feria Internacional del Libro de Lima se presentó Presencia italiana en el arte peruano del siglo $X X$ de la historiadora de arte Nanda Leonardini Herane, publicación que testimonia la importancia de la colonia y los artistas italianos en el desarrollo de la cultura y el arte en el Perú a lo largo del siglo XX. Los comentarios estuvieron a cargo de Humberto Rodríguez Pastor y Alfonso Castrillón Vizcarra.

El 9 de agosto en la Galería de Artes Visuales, se presentó Globalización, identidad y resistencia, libro de Blanca Gutiérrez que trata sobre el "otro derecho al patrimonio histórico» en la ciudad del Cusco. Los comentarios estuvieron a cargo de Alfonso Castrillón Vizcarra y fue organizado por el Instituto de Investigaciones Museológicas.

De tradiciones y gestiones: El legado de Ricardo Palma Soriano a la sociedad peruana, exposición documental realizada por los cincuenta años de aniversario de la Universidad Ricardo Palma y como homenaje por el centenario del fallecimiento del tradicionista. La exposición estuvo bajo la curaduría de CURA soluciones museográficas y buscó resaltar los aportes que dejó "El Bibliotecario Mendigo" a la sociedad, no solo como literario sino también como gestor de la Biblioteca Nacional del Perú, el auspicio que le brindó a Julio C. Tello, padre de la arqueología peruana; su intervención en el cuidado e interpretación a las acuarelas de Francisco "Pancho" Fierro, entre otras de sus múltiples acciones. La exposición estuvo del 13 de septiembre al 27 de octubre en la Galería de Artes Visuales de la Universidad Ricardo Palma.

El 17 de octubre se llevó a cabo la Romería al mausoleo de Don Ricardo Palma ubicado en el Museo Cementerio Presbítero Maestro, con motivo del celebrarse el centenario del fallecimiento del insigne tradicionista. El homenaje estuvo organizado por el Rectorado y el Instituto de Investigaciones Museológicas y Artísticas y contó con el apoyo de la Oficina Central de Extensión Cultural y Proyección Social, la Oficina de Imagen Institucional y la Oficina de Administración y Mantenimiento de la Universidad Ricardo Palma. 
Del 5 al 20 de noviembre se llevó acabo en la Galería de Artes Visuales de la Universidad Ricardo Palma la VII edición de Lima Design Week, evento que combina arte, cultura y diferentes expresiones de diseño con el objetivo de difundir la importancia del diseño como un enlace para el desarrollo social y la innovación. Y en el que se desarrollaron diversas actividades como talleres, conferencias, rutas de diseño y exposiciones donde el público local e internacional descubrió una variedad de diseños multidisciplinarios: diseño industrial, diseño de interiores, diseño de productos y diseño de moda.

Los días 20, 21 y 22 de noviembre, se llevó a cabo el VI Seminario Internacional sobre Arte público en Latinoamérica, en coordinación por el Instituto de Investigaciones Museológicas y Artísticas de la Universidad Ricardo Palma y el Grupo de Estudio de Arte Público en Latinoamérica (GEAP). El seminario tuvo como objetivo formar una red de investigadores que permita conocer estas prácticas desde diferentes perspectivas, con una visión interdisciplinaria, y crear un ámbito de reflexión, debate y difusión al servicio de investigadores que aborden el arte público según intereses diversos: patrimoniales, históricos, iconográficos, artísticos, de conservación y restauración, entre otros.
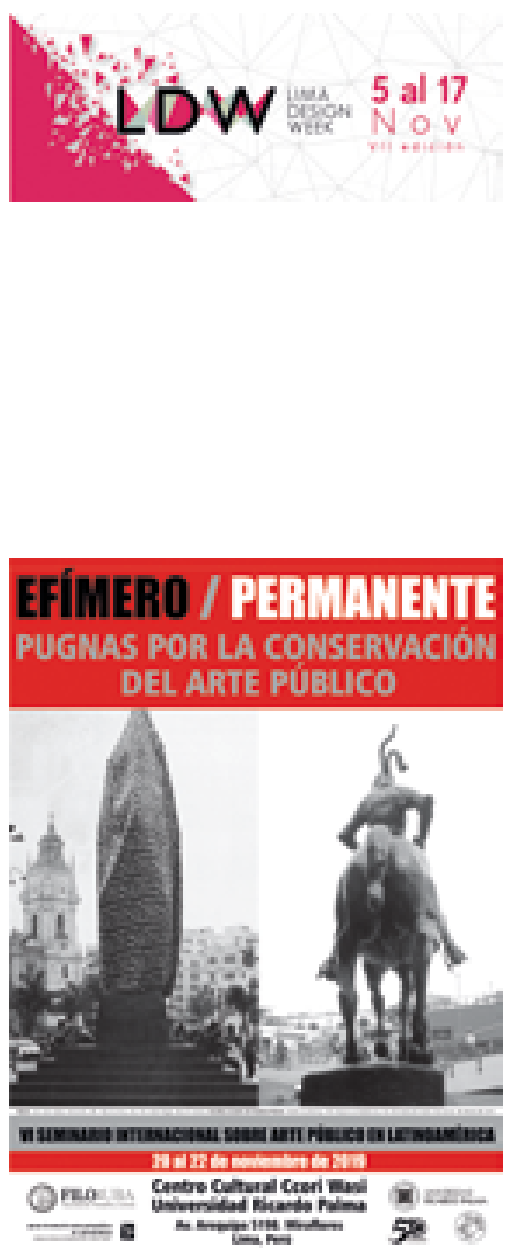